\title{
Annals of Hematology
}

\section{Impact of comorbidities and body mass index in patients with myelofibrosis treated with ruxolitinib}

--Manuscript Draft--

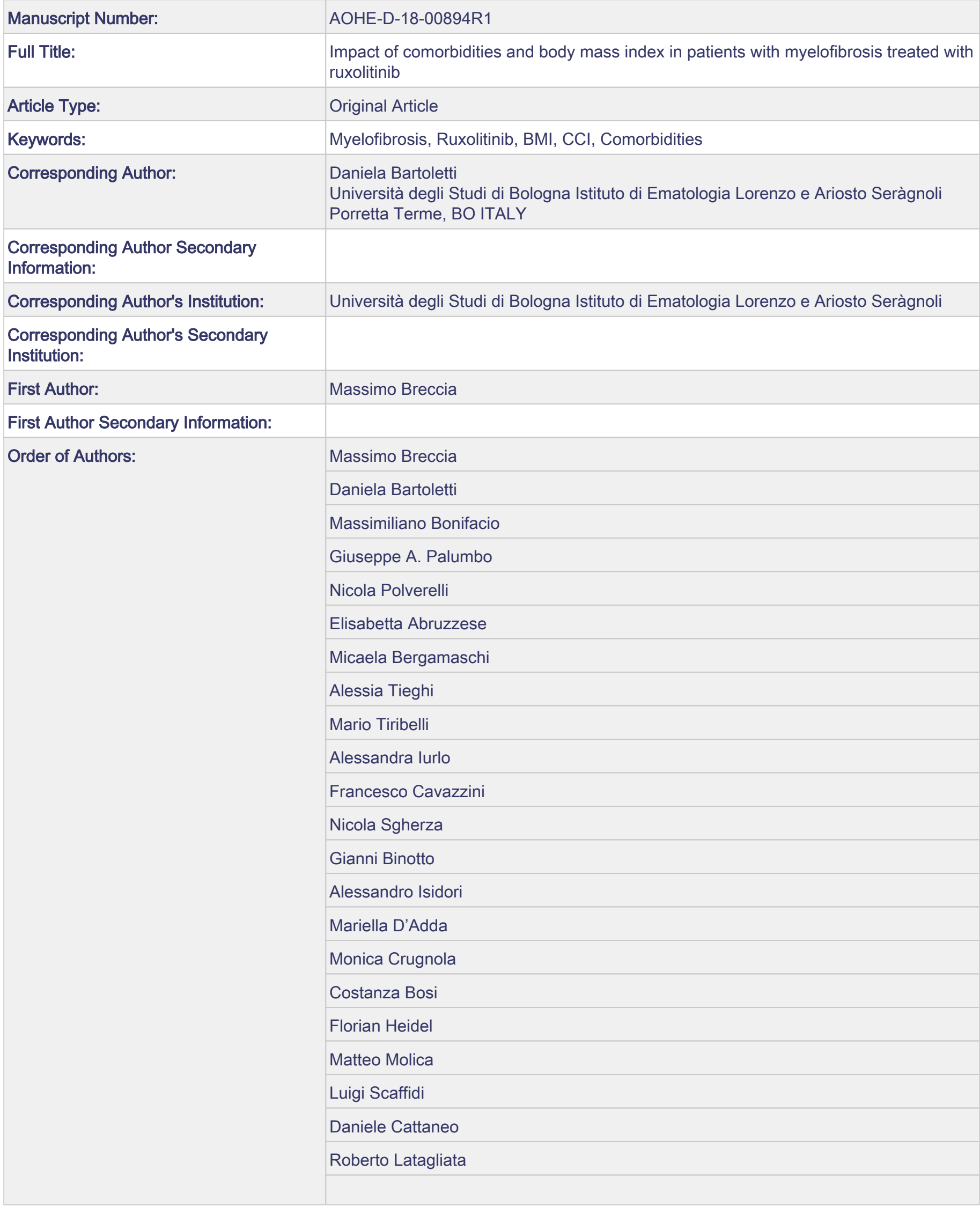




\begin{tabular}{|c|c|}
\hline \multicolumn{2}{|c|}{\begin{tabular}{l|l} 
Giuseppe Auteri
\end{tabular}} \\
\hline & Roberto M. Lemoli \\
\hline & Renato Fanin \\
\hline & Domenico Russo \\
\hline & Franco Aversa \\
\hline & Antonio Cuneo \\
\hline & Gianpietro Semenzato \\
\hline & Lucia Catani \\
\hline & Michele Cavo \\
\hline & Nicola Vianelli \\
\hline & Robin Foà \\
\hline & Francesca Palandri \\
\hline \multicolumn{2}{|c|}{ Order of Authors Secondary Information: } \\
\hline \multicolumn{2}{|l|}{ Funding Information: } \\
\hline Abstract: & $\begin{array}{l}\text { Background } \\
\text { Comorbidities defined by the Charlson comorbidity index }(\mathrm{CCl}) \text { and body mass index } \\
\text { (BMI) are significantly associated with outcome in patients who receive continuous } \\
\text { treatment with tyrosine kinase inhibitors. } \\
\text { Methods } \\
\text { We evaluated the impact of } \mathrm{CCl} \text { and BMI on responses, drug-related toxicities and } \\
\text { outcome in a cohort of } 402 \text { patients with myelofibrosis (MF) treated with ruxolitinib in } 23 \\
\text { European Hematology Centers. } \\
\text { Results } \\
\text { Comorbidities were evaluable in all } 402 \text { patients. A higher (>3) CCI did not correlate } \\
\text { with a lower spleen reduction at any time ( } p=0.68 \text { ) or symptoms' response ( } \mathrm{p}=0.11 \text { ), } \\
\text { but influenced the onset of anemia (all grades) any time and during the first } 3 \text { months } \\
\text { of treatment and later ( } p=0.04 \text { and } p=0.03 \text {, respectively). BMI was evaluable in } 380 \\
\text { patients and did not correlate with differences in spleen and symptoms response } \\
\text { ( } p=0.57 \text { and } p=0.49, \text { respectively). A higher CCI and a lower BMl correlated also with a } \\
\text { reduced overall survival ( } p<0.001 \text { and } p=0.02 \text {, respectively). The achievement of a } \\
\text { spleen response at } 6 \text { months could counterbalance the negative impact of } \\
\text { comorbidities, while patients who were underweight when starting ruxolitinib and did } \\
\text { not achieve a spleen response at } 6 \text { months were projected to the worse outcome. } \\
\text { Conclusions } \\
\text { In MF patients treated with ruxolitinib, BMI and comorbidities did not influence the } \\
\text { achievement of spleen/symptom responses, but they contributed to the early } \\
\text { identification of patients who deserve a strict monitoring during treatment. }\end{array}$ \\
\hline Response to Reviewers: & $\begin{array}{l}\text { Reviewer \#1 } \\
\text { Interesting analyses on outcome of MF patients also correlating comorbidities and BMI. } \\
\text { Some points to discuss: } \\
\text { - It should be discussed if patients with MF per se should be counted in the CCI with } 2 \\
\text { points (Definition leukemia: Leukemia - malignancy due to abnormal function of the } \\
\text { bone marrow and the other blood forming organs with an increased number of } \\
\text { immature and/or abnormal leucocytes) at least for patients with advanced phases. } \\
\text { We agree with the Reviewer that all patients carry the same hematological }\end{array}$ \\
\hline
\end{tabular}


malignancy (MF); however, if we add 2 points for each patient (applying the term "leukemia" to all MF patients), the results of the present analysis would not change. Therefore, we have assigned 2 points only in case of additional malignancy prior to ruxolitinib start. This has been specified in the Material and Methods Section.

- For the BMI categories it would be interesting how the values were set. Was there any approach to have the analyses done by minimal p-value approach?

Minimal $p$-value approach was not used for the division in categories according to BMI. We stratified patients into 3 categories according to BMI, with the aim of comparing underweight, normal-weight and overweight patients. The first cut-off was however set at 21.9 in order to include the first quartile (n.96) of patients, to avoid having small numbers (only $3.2 \%$ pts had a BMI $<18.5$ ) thereby preventing substantial losses in statistical power, while the second cut-off was set at 25, in order to isolate the overweight group of patients, in compliance with the WHO stratification criteria. This was specified in the Result Section (Impact of comorbidities and BMI on response to ruxolitinib).

- How many patients were transplanted and were they censored for survival analyses?

Overall, $17(4.2 \%)$. patients were submitted to allogeneic stem cell transplant after ruxolitinib failure. These patients were censored at the time of transplant for survival analysis.

This has been specified in the Result Section (Impact of comorbidities and BMI on outcome).

- The figures are difficult to read in the current outline (the differences in Grey are margial).

Ok, figures have been edited in order to improve their readability also in black and white.

Reviewer \#2

This multicenter study analyzed the impact of comorbidities (defined according to the Charlson comorbidity index , $\mathrm{CCl}$ ) and body mass index (BMI) on responses, drugrelated toxicities and outcome in a cohort of 402 patients with myelofibrosis (MF) treated with ruxolitinib. Main results showed that neither comorbidities nor BMI influence the probability of achieving a response on spleen or systemic symptoms during ruxolitinib treatment. Severe anemia, thrombocytopenia and the incidence of infections were not influenced by $\mathrm{CCl}$ and $\mathrm{BMI}$. As expected, a higher $\mathrm{CCl}$ and a lower $\mathrm{BMI}$ correlated with a reduced overall survival. The Authors concluded that comorbidities and BMI do not seem to be a contraindication for ruxolitinib therapy in MF patients.

Major scientific issues

1. The issue of anemia should be better addressed. Anemia was already present at ruxolinib start (tab. 1) and "patients with an increased burden of comorbidities presented more frequently anemia at baseline" (Discussion, para 2, line 7). Therefore, the statement that "comorbidities influenced the onset of ruxolitinib-related anemia (all grades) " (Abstract, results, lines 2-3 and page 4, toxicity, para 2, first line) is confusing, since anemia was already present and was not ruxolitinib-related. Accordingly, the hemoglobin level at baseline could be sufficient for 'the early identification of patients who deserve a strict monitoring during treatment' without using the $\mathrm{CCl}$; thus, the last sentence of the Abstract is futile.

In the first version of the paper, drug-induced anaemia was defined according to National Cancer Institute Common Toxicity Criteria for Adverse Events (NCI-CTCAE) v 4.0 (https://www.eortc.be/services/doc/ctc/CTCAE_4.03_2010-06-

14_QuickReference_5x7.pdf). Patients that were transfusion-dependent before the start of ruxolitinib therapy were not evaluable for drug-related anaemia. However, 
patients with baseline MF-related anemia (haemoglobin levels below $12 \mathrm{~g} / \mathrm{dl}$ in absence of transfusion requirement) were included in the analysis.

We agree with the Reviewer that this analysis may be confusing.

Therefore, firstly, we have repeated the analysis taking into account only drug-induced anemia, defined as increasing in anemia grade with respect to baseline levels. This analysis failed to show any impact of $\mathrm{BMI}$ and $\mathrm{CCl}$ on drug-induced anemia. These results are reported in the Results section and in Table 2, which has been revised. Secondly, we have analysed the incidence of anemia only in the 132 patients that started ruxolitinib without anemia (i.e: with a haemoglobin level $\geq 12 \mathrm{~g} / \mathrm{dl}$ in absence of transfusion requirement). Here, we demonstrated that patients with $\mathrm{CCl} \geq 3$ had a higher incidence of ruxolitinib-induced grade- $\geq 2$ anemia at 3 months and at any time during therapy. Therefore, our statement that comorbidities influenced the onset of ruxolitinibrelated anemia is valid for ruxolitinib-induced grade- $\geq 2$ anemia, These results are now reported in the Results section and in a new Table 3.

2. The statistical analysis reported in fig. $2 a$ and $b$ is unclear. At which comparisons the $\mathrm{p}$ values are applied?

The $p$-values in Figures $2 \mathrm{a}$ and $2 \mathrm{~b}$ are results of Log-rank tests and represent the overall difference between the Kaplan-Meier curves.

We have expanded the analysis with pair-wise Log-rank tests between the curves, and the $p$-values of the single log-rank pairwise survival comparisons are reported in the description of Figure 2 and confirm that the categories that significantly differ from the others (and that majorly impact on the overall difference between the categories) are represented, in Figure 2a, by the group of patients with a spleen response and with lower $\mathrm{CCl}$, that have a significantly improved Overall Survival compared to the others, and, in Figure $2 \mathrm{~b}$, by the patients with no spleen response and a lower BMI, that have a significantly worse Overall Survival compared to the others.

3. Some conclusions are not supported by data, such as "The cut-off of $\mathrm{CCl}(<3)$ could be of help to identify patients who may deserve a treatment with ruxolitinib...

(Discussion, para 2, lines 5-6)". Actually, the results did not show differences in the response to ruxolitinib according to $\mathrm{CCl}$.

Ok. This sentence actually referred to the result that the patients with both spleen response and $\mathrm{CCl}<3$ are projected to longer survival (Figure 2a) and may therefore represent the best target population for ruxolitinib therapy.

The sentence has been rephrased in the text and is now clearer.

Other points

1. Several repetitions should be avoided. For example, the information that 'BMI was evaluated in 380 patients' is given four times in the paper.

Ok, repetitions have been deleted.

2. Ref. 25 should be corrected.

Ok, ref 25 has been edited. 
Table 1. Patients' characteristics at ruxolitinib start. Post-PV MF: Post-Polycythemia vera Myelofibrosis; Post-ET MF: Post-Essential Thrombocythemia Myelofibrosis. DIPSS: Dynamic International Prognostic Score System.

\begin{tabular}{|c|c|}
\hline Characteristics & Patients (n. 402) \\
\hline Male sex, n. (\%) & $233(58.0 \%)$ \\
\hline Primary MF, n. (\%) & $212(52.7 \%)$ \\
\hline Post-PV MF, n. (\%) & $117(29.1 \%)$ \\
\hline Post-ET MF, n. (\%) & $73(18.2 \%)$ \\
\hline \multicolumn{2}{|l|}{ DIPSS risk score, on 363 evaluable patients, n. (\%) } \\
\hline Intermediate-1 & $156(43.0 \%)$ \\
\hline Intermediate-2 & $183(50.4 \%)$ \\
\hline High & $24(6.6 \%)$ \\
\hline Median hemoglobin, g/dl (range) & $10.7(4.7-16.7)$ \\
\hline Transfusion dependence, n. (\%) & $102(25.4 \%)$ \\
\hline Median platelet count, $\times 10^{9} / 1$ (range) & $248.5(32.9-1632)$ \\
\hline Palpable spleen, n. (\%) & $386(96.0 \%)$ \\
\hline Spleen $\geq 10 \mathrm{~cm}, \mathrm{n} .(\%)$ & $252(62.7 \%)$ \\
\hline Median Body Mass Index (BMI), n. (\% on 380 evaluable patients) & $23.9(15.5-33.3)$ \\
\hline BMI <21.9 (first quartile) & $96(25.3 \%)$ \\
\hline BMI 21.9-24.9 (normal weight) & $141(37.1 \%)$ \\
\hline BMI $\geq 25$ (overweight) & $143(37.6 \%)$ \\
\hline Unfavorable karyotype, n. (\% on 213 evaluable patients) & $16(7.5 \%)$ \\
\hline JAK $2^{\text {V617F }}$, n. (\% on 321 evaluable patients) & $282(87.9 \%)$ \\
\hline CALR, n. (\% on 321 evaluable patients) & $26(8.1 \%)$ \\
\hline MPL, n. (\% on 321 evaluable patients) & $1(0.3 \%)$ \\
\hline Triple negative, n. (\% on 321 evaluable patients) & $12(3.7 \%)$ \\
\hline Median time from MF diagnosis to ruxolitinib start, months & $16.1(0-290.5)$ \\
\hline \multicolumn{2}{|l|}{ Ruxolitinib starting dose, n._(\%) } \\
\hline $5 \mathrm{mg}$ BID & $55(13.7 \%)$ \\
\hline $10 \mathrm{mg}$ BID & $38(9.5 \%)$ \\
\hline $15 \mathrm{mg}$ BID & $103(25.6 \%)$ \\
\hline $20 \mathrm{mg}$ BID & $206(51.2 \%)$ \\
\hline Median follow-up from ruxolitinib start, months (range) & $23.1(1.1-68.0)$ \\
\hline
\end{tabular}


Impact of comorbidities and body mass index in patients with myelofibrosis treated with ruxolitinib

Massimo Breccia ${ }^{1}$, Daniela Bartoletti ${ }^{2}$, Massimiliano Bonifacio ${ }^{3}$, Giuseppe A. Palumbo ${ }^{4}$, Nicola Polverelli $^{5}$, Elisabetta Abruzzese ${ }^{6}$, Micaela Bergamaschi ${ }^{7}$, Alessia Tieghi ${ }^{8}$, Mario Tiribelli ${ }^{9}$, Alessandra Iurlo ${ }^{10}$, Francesco Cavazzini ${ }^{11}$, Nicola Sgherza ${ }^{12}$, Gianni Binotto ${ }^{13}$, Alessandro Isidori ${ }^{14}$, Mariella D'Adda ${ }^{15}$, Monica Crugnola ${ }^{16}$, Costanza Bosi ${ }^{17}$, Florian Heidel ${ }^{18}$, Matteo Molica ${ }^{1}$, Luigi Scaffidi $^{3}$, Daniele Cattaneo ${ }^{10}$, Roberto Latagliata ${ }^{1}$, Giuseppe Auteri ${ }^{2}$, Roberto M. Lemoli ${ }^{7}$, Renato Fanin $^{9}$, Domenico Russo ${ }^{5}$, Franco Aversa ${ }^{16}$, Antonio Cuneo ${ }^{11}$, Gianpietro Semenzato ${ }^{13}$, Lucia Catani $^{2}$, Michele Cavo ${ }^{2}$, Nicola Vianelli ${ }^{2}$, Robin Foà ${ }^{1}$, Francesca Palandri ${ }^{2}$

${ }^{1}$ Division of Cellular Biotechnologies and Hematology, University Sapienza, Rome, Italy; ${ }^{2}$ Institute of Hematology "L. and A. Seràgnoli”, Sant'Orsola-Malpighi University Hospital, Bologna Italy; ${ }^{3}$ Department of Hematology, University of Verona, Verona, Italy; ${ }^{4}$ Division of Hematology, AOU "Policlinico-V. Emanuele", University of Catania; ${ }^{5}$ Unit of Blood Diseases and Stem Cells Transplantation, Department of Clinical and Experimental Sciences, University of Brescia, ASST Spedali Civili of Brescia; ${ }^{6}$ Division of Hematology, Ospedale S. Eugenio, Roma; ${ }^{7}$ Clinic of Hematology, Department of Internal Medicine (DiMI), IRCCS AOU San Martino-IST, Genova; ${ }^{8}$ Division of Hematology, Azienda Ospedaliera-IRCSS Arcispedale Santa Maria Nuova, Reggio Emilia; ${ }^{9}$ Division of Hematology and BMT, Department of Medical Area, University of Udine; ${ }^{10}$ Hematology Division, IRCCS Ca' Granda - Maggiore Policlinico Hospital Foundation, University of Milan; ${ }^{11}$ Division of Hematology, University of Ferrara; ${ }^{12}$ Division of Hematology, Casa Sollievo Sofferenza, San Giovanni Rotondo; ${ }^{13}$ Unit of Hematology and Clinical Immunology, University of Padova; ${ }^{14}$ Hematology and Stem Cell Transplant Center, AORMN Hospital, Pesaro; ${ }^{15}$ Division of Hematology, ASST Spedali Civili di Brescia; ${ }^{16}$ Division of Hematology, Azienda Ospedaliero-Universitaria di Parma; ${ }^{17}$ Division of Hematology, Piacenza; ${ }^{18}$ Internal Medicine II, Hematology and Oncology, Friedrich-Schiller-University Medical Center, Jena, Germany

Running title: Impact of comorbidities and BMI in ruxolitinib-treated patients with myelofibrosis

Keywords: Myelofibrosis, Ruxolitinib, BMI, CCI, Comorbidities

\author{
Corresponding Author: \\ Massimo Breccia, MD \\ Hematology \\ Department of Cellular Biotechnologies and Hematology \\ Sapienza University \\ Via Benevento 6, 00161 Rome, Italy \\ Tel. +3906857951 \\ Fax +390644241984 \\ e-mail: breccia@bce.uniroma1.it
}




\section{Abstract}

Background. Comorbidities defined by the Charlson comorbidity index (CCI) and body mass index (BMI) are significantly associated with outcome in patients who receive continuous treatment with tyrosine kinase inhibitors.

Methods. We evaluated the impact of CCI and BMI on responses, drug-related toxicities and outcome in a cohort of 402 patients with myelofibrosis (MF) treated with ruxolitinib in 23 European Hematology Centers.

Results. Comorbidities were evaluable in all 402 patients. A higher $(\geq 3) \mathrm{CCI}$ did not correlate with a lower spleen reduction at any time $(\mathrm{p}=0.68)$ or symptoms' response $(\mathrm{p}=0.11)$, but influenced the onset of anemia during the first 3 months of treatment and later $(\mathrm{p}=0.02$ and $\mathrm{p}=0.03$, respectively) in patients without anemia baseline. BMI was evaluable in 380 patients and did not correlate with differences in spleen and symptoms response ( $\mathrm{p}=0.57$ and $\mathrm{p}=0.49$, respectively). A higher CCI and a lower BMI correlated also with a reduced overall survival $(\mathrm{p}<0.001$ and $\mathrm{p}=0.02$, respectively). The achievement of a spleen response at 6 months could counterbalance the negative impact of comorbidities, while patients who were underweight when starting ruxolitinib and did not achieve a spleen response at 6 months were projected to the worse outcome.

Conclusions. In MF patients treated with ruxolitinib, BMI and comorbidities did not influence the achievement of spleen/symptom responses, but they contributed to the early identification of patients who deserve a strict monitoring during treatment.

\section{Introduction}

In the last few years, several reports have demonstrated the impact of comorbidities on survival in chronic myeloid leukemia (CML) patients treated with tyrosine kinase inhibitors (TKIs). Indeed, the German group has shown in a large CML series that a higher Charlson comorbidity index $(\mathrm{CCI})$ at baseline significantly correlated with reduced survival regardless of the response achieved during imatinib therapy [1]. In addition, comorbidities predicted worse prognosis in patients with primary myelofibrosis [2]. Together with comorbidities, also body mass index may be relevant in prognosis of patients with cancer [3]. According to WHO criteria, people may be stratified into four categories: underweight (BMI <18.5), normal weight (18.5-25), overweight (25$30)$ and obese $(\geq 30)$ [4]. Increased BMI was associated with delayed cytogenetic and major molecular responses to imatinib in CML patients [5].

Ruxolitinib is the first JAK1/JAK2 inhibitor approved for the treatment of splenomegaly and symptoms associated with myelofibrosis (MF). The prospective COMFORT 1 and COMFORT 2 studies randomized MF patients to receive either ruxolitinib or placebo and best available therapies, respectively, demonstrating the superiority of ruxolitinib in terms of reduction of splenomegaly, amelioration of symptoms and improvement of quality of life [6, 7]. A longer follow-up of the COMFORT studies also documented a survival advantage of patients treated with ruxolitinib compared to placebo and best available treatment $[8,9]$. The impact of comorbidities has not been detailed systematically in the COMFORT studies or the JUMP trial [10] and only $13 \%$ of patients assigned to ruxolitinib showed a performance score ECOG $>2$ in the COMFORT-II trial [8]. Therefore, a clear benefit of ruxolitinib for patients with impaired clinical conditions has never been 
demonstrated and the European LeukemiaNet/SIE panel has suggested to avoid the drug in frail patients [11].

Here, we retrospectively assessed the impact of comorbidities and BMI on clinical responses, overall survival and maintenance of ruxolitinib dose in a large cohort of MF patients.

\section{Methods}

An electronic database was established to collect clinical, molecular and laboratory data on MF patients treated with ruxolitinib in 23 European Hematology Centers as previously described [12]. Between June 2011 and November 2016, data on 462 consecutive MF patients were retrospectively collected. Data cut-off was January 2018. Two-hundred and thirty-four patients were enrolled in the JUMP trial (ClinicalTrials.gov Identifiers: NCT01493414) and 168 patients received ruxolitinib as compassionate or commercial use/off-study according to the standard clinical practice.

A diagnosis of primary myelofibrosis (PMF) and post-essential thrombocythemia/polycythemia vera myelofibrosis (post-ET/post-PV MF) was made according to the WHO 2008 [13] or the International Working Group on Myelofibrosis Research and Treatment (IWG-MRT) criteria [14]. Bone marrow fibrosis was graded according to the European Consensus Grading System [15]. Evolution into blast phase was based on the WHO classification [16]. Molecular tests for detection of JAK2, MPL and CALR mutations and cytogenetic analysis were carried out as described elsewhere [17]. Ruxolitinib was administrated according to the prescribing information. Spleen and symptoms' responses have been defined according to the International Working Group on Myelofibrosis Research and Treatment / European Leukemia Net (IWGMRT/ELN) criteria [18]. Symptoms' response was assessed by changes in the Myeloproliferative Neoplasm Symptom Assessment Form total symptoms score (TSS) [19].

Anemia and thrombocytopenia were graded according to National Cancer Institute Common Toxicity Criteria for Adverse Events (NCI-CTCAE) v 4.0 (https://www.eortc.be/services/doc/ctc/ CTCAE_4.03_2010-06-14_QuickReference_5x7.pdf). Drug-induced anemia and thrombocytopenia were defined as increasing in anemia/thrombocytopenia grade with respect to baseline levels. Patients that were transfusion-dependent before the start of ruxolitinib therapy were not evaluable for subsequent anemia.

BMI was calculated at the time of start of ruxolitinib treatment. BMI was defined as the individual's body weight in $\mathrm{Kg}$ divided by the square of his/her height, which produces a unit of measure of $\mathrm{Kg} / \mathrm{m} 2$.

Comorbidities were recorded at the time of the start of ruxolitinib and classified according to the CCI [20]. Comorbidities were evaluated at baseline, before ruxolitinib treatment, by the medical staff. All information about concomitant diseases and drug usage were recorded in each case history and thereafter used for this retrospective evaluation. As reported, CCI is a list of 19 comorbid conditions: each condition has a weight assigned from 1 to 6 , which is derived from the relative risk estimates of a proportional hazard regression model using clinical data. For the condition "leukemia-malignancy", 2 points were assigned only in case of occurrence of an additional malignancy to MF prior to ruxolitinib start.

The study was approved by the Institutional Review Board of each Institution and was conducted according to the Helsinki declaration. Clinical and laboratory parameters were evaluated both at diagnosis and at the start of ruxolitinib. 


\section{Statistical analysis}

Categorical variables have been summarized by their median and range or mean and standard deviation, according to the statistical analysis performed, and categorical variables by count and relative frequency $(\%)$ of each category. Comparisons of continuous variables between groups of patients were carried out using the Wilcoxon-Mann-Whitney rank-sum test or the Student's t-test, when compared between two groups, or the ANOVA or Kruskal-Wallis tests, when compared between 3 groups, and the association between categorical variables (2-way tables) was tested by the Fisher exact test or $\chi 2$, as appropriate. Overall Survivals (OS) were performed using Kaplan-Meier analysis, from the start of ruxolitinib to death or last contact, and compared by the log-rank test, also used for pairwise comparisons when considering multiple categories. Progression-free survival (PFS) took into account death from any cause and progression to blast phase. When calculating OS and PFS from the start of ruxolitinib, the survival analysis was adjusted for left truncation ("delayed entry"). When adjusting for the Dynamic International Prognostic Score System (DIPSS) category at treatment, OS and PFS were calculated using the Cox proportional hazards multivariate regression model. The cumulative incidence of infections was calculated considering death as competing risk, according to the model of Fine and Gray. All reported $\mathrm{p}$ values are two-sided, and $\mathrm{p}$ values $<0.05$ were considered statistically significant. All statistical analyses were performed with STATA15.

\section{Results}

\section{Study cohort}

Between June 2011 and April 2016, 462 MF patients were treated with ruxolitinib in the participating Centers. Of the whole cohort, a total of 402 patients were evaluable for CCI and were included in this study. Three hundred and eighty patients $(94.5 \%)$ had also data on BMI at the start of treatment.

At ruxolitinib start, the median age was 64 years (range 39-89) with a male prevalence (52.4\%). Diagnosis was PMF in 212 patients (52.7\%), post-PV MF in 117 patients $(29.1 \%)$ or postET MF in $18.2 \%$ of cases. The IPSS at ruxolitinib start was intermediate- 1 in $15.7 \%$ of cases, intermediate- 2 in $46.5 \%$ or high in $37.8 \%$. The median follow-up from MF diagnosis to the last contact was 3.8 years (range 0.2-34.8) and the median exposure to ruxolitinib exposure 23.1 months (range, 1.1-68) (Table 1).

Among the 402 evaluable patients, $198(49.2 \%)$ had no comorbidities, 128 (31.8\%) had a CCI $1-2$ and $76(40.7 \%)$ a CCI $\geq 3$, with a median of 1 comorbidity per patient (range $0-8)$. The most common comorbidities were peripheral vascular disease (14\%), previous solid tumor (13.5\%), diabetes $(10 \%)$ and liver disease $(9.6 \%)$. Compared to patients with CCI=0, patients with CCI 1-2 and $\geq 3$ were more frequently in the intermediate-2 DIPPS category $(\mathrm{p}=0.03)$, with a hemoglobin level $<10 \mathrm{gr} / \mathrm{dl}(33.8 \%$ in $\mathrm{CCI}=0,43.7 \%$ in $\mathrm{CCI}=1-2$ and $56.6 \%$ in $\mathrm{CCI} \geq 3, \mathrm{p}=0.005)$ and transfusion dependence ( $20 \%$ vs $28.1 \%$ and $34.2 \%, \mathrm{p}=0.04$ ).

Overall, 12 patients (3.2\%) had a BMI <18.5, $225(59.2 \%)$ were normal weighted, 123 (32.4\%) a BMI between 25 and 30 while 20 patients (5.2\%) were obese. Median BMI was 23.9 (range: 15.5-33.3). Overweight patients (BMI $\geq 25)$ were more frequently males $(\mathrm{p}=0.001)$, belonged more frequently to the intermediate-1 DIPSS category, and had less anemia: a hemoglobin 
value $<10 \mathrm{gr} / \mathrm{dl}$ at presentation was found in $45.0 \%$ of patients with a BMI $<25$ and $33.8 \%$ with a

BMI $\geq 25$ ( $p=0.03$ ). The burden of symptoms, in terms of presence of constitutional symptoms and average 10-item TSS was not significantly influenced by CCI and BMI.

\section{Impact of comorbidities and BMI on response to ruxolitinib}

Overall, 354 patients were evaluable for spleen response and 379 for symptoms response according to the IWG-MRT criteria. A total of $138(39.0 \%)$ and $309(81.5 \%)$ patients achieved a spleen or a symptom response at any time during therapy, respectively. More specifically, at 3 and 6 months $100(28.2 \%)$ and $122(37.9 \%)$ out of 354 and 322 evaluable patients were in spleen response, while $277(73 \%)$ and $263(82.1 \%)$ out of 379 and 320 evaluable patients were in symptoms response, respectively.

By stratifying patients into 3 categories with different $\mathrm{CCI}(\mathrm{CCI}=0$, $\mathrm{CCI} 1-2$ and $\mathrm{CCI} \geq 3$ ), a higher CCI did not correlate with a lower spleen response at any time (achieved by $37.6 \%, 38.5 \%$ and $43.7 \%$ of patients with CCI $=0,1-2$ and $\geq 3$, respectively, $\mathrm{p}=0.68$ ), or symptoms response at any time (achieved by $77.3 \%, 85.9 \%$ and $84.9 \%$ in patients with $\mathrm{CCI}=0,1-2$ and $\geq 3$, respectively, $\mathrm{p}=0.11$ ). The rates of spleen and symptoms responses were also comparable at 3 and 6 months across the three CCI groups.

Patients were also subdivided into three categories according to a BMI below the first quartile ( $<21.9$, n.96), a BMI between 21.9 and 24.9 (normal weight, n. 142) and BMI $\geq 25$ (overweight, n. 142). BMI categories were not significantly associated with differences in spleen response (achieved by $40.7 \%, 35.2 \%$ and $41.3 \%$ of patients with a BMI $<21.9,21-24.9$ and $\geq 25$, respectively, $\mathrm{p}=0.57$ ), or symptom response (achieved by $79.3 \%, 79.7 \%$ and $84.6 \%$ of patients, respectively, $\mathrm{p}=0.49$ ) at any time. Also, the rates of spleen and symptoms responses were comparable across the three groups of patients both at $3(\mathrm{p}=0.85$ and $\mathrm{p}=0.32)$ and 6 months $(\mathrm{p}=0.73$ and $\mathrm{p}=0.63$ ).

Average starting and overall ruxolitinib doses were comparable across both $\mathrm{CCI}(\mathrm{p}=0.78)$ and BMI $(\mathrm{p}=0.37)$ groups.

\section{Impact of comorbidities and BMI on toxicity to ruxolitinib}

Overall, $229(75.6 \%)$ of 303 evaluable patients, who were not transfusion-dependent at the start of ruxolitinib, experienced an increase in anemia grade; comorbidities and BMI did not influence the increase of ruxolitinib-related anemia grade over time (Table 2). However, the incidence of ruxolitinib-induced grade $\geq 2$ anemia in the 132 patients that started ruxolitinib without anemia (i.e: with a haemoglobin level $\geq 12 \mathrm{~g} / \mathrm{dl}$ in absence of transfusion requirement) was significantly higher in patients with $\mathrm{CCI} \geq 3$ (Table 3 ).

Thrombocytopenia of any grade at any time was observed in 204 of the 394 evaluable patients (grade 3-4 in 10.8\%) and was never associated with CCI and BMI (Tables 2 and 3).

Infections grade $\geq 2$ occurred in 120 (29.9\%) patients, after a median time form ruxolitinib start of 5.9 months (range: 1-60.9). More specifically, infections were: pneumonia in $25.8 \%$ of cases (grade 2 in 11 patients, grade 3 in 14 and grade 4-5 in 6), bronchitis in 15\% of cases (grade 2 in 16 patient and grade 3 in two), gastrointestinal infections in $8.3 \%$ of cases (grade 2 in 4 patients, grade 3 in 5 and grade 4 in 1), urinary tract infections in 8.3\% of cases (grade 2 in 9 patients and 
grade 3 in 1), Herpesvirus Infections in 10\% of cases (grade-2 Herpes Simplex infections in 6 patients and grade- 2 and grade- 3 Herpes zoster infections in 5 and 1 patient, respectively), cutaneous infections in 5.8\% of cases (grade-2 in 6 patients and grade- 3 in 1), upper airways infections in $7.7 \%$ of cases (grade- 2 in 8 patients and grade-3 in 1), fever in $15 \%$ of cases (grade- 2 in 13 patients and grade $3-4$ in 5), sepsis in $1.7 \%$ of cases (one grade- 3 and grade-4 in 1). In addition, grade- 2 tuberculosis, grade- 2 eye infection and grade- 2 renal infection were recorded in 1 patient each. After adjustment for risk of death, the cumulative incidence of infections during treatment was not influenced by CCI and BMI stratification ( $\mathrm{p}=0.27$ and $\mathrm{p}=0.95$, respectively).

\section{Impact of comorbidities and BMI on outcome}

Overall, $17(4.2 \%)$. patients were submitted to allogeneic stem cell transplant after ruxolitinib failure. These patients were censored at the time of transplant for survival analysis.

Accounting for left-truncation, overall and progression-free survival differed significantly between the different CCI groups ( $\mathrm{p}=0.01$ and 0.04 , respectively). CCI maintained its prognostic value even when adjusted for DIPSS ( $\mathrm{p}<0.001$ and 0.001 , respectively) (Figure 1a and 1b). As in the CCI stratification, also the BMI correlated with DIPSS-adjusted OS $(\mathrm{p}=0.003)$ and PFS $(\mathrm{p}=0.003)$ (Figure 1c and 1d) after left truncation.

A landmark Kaplan-Meier survival analysis, from 6 months from ruxolitinib start onwards, was performed to compare patients according to response to ruxolitinib at 6 months and CCI. Logrank tests showed that patients with an IWG-MRT-defined spleen response and a CCI $<3$ had the best OS compared to all the other categories (Figure 2a). Notably, in patients with a comparable $\mathrm{CCI}$ below 3, the achievement of a spleen response at 6 months significantly improved OS. Logrank tests also showed that patients who did not obtain a response and with a BMI $<21.9$ had the worse outcome (Figure $2 b$ ). Here, achieving a spleen response could significantly improve the outcome of patients starting treatment while underweight.

\section{Discussion}

Comorbidities influence the prognosis of patients affected by cancers and impact on treatment decisions [2, 21-23]. While an accurate evaluation of comorbidities and nutritional status is now part of the routine baseline assessment of CML patients, these parameters have never been investigated in depth in patients with MF treated with ruxolitinib.

Our analysis in a large series of MF patients homogeneously treated with ruxolitinib in the clinical practice showed that baseline comorbidities, such as in other diseases, have a role in predicting survival, but do not influence the probability of achieving a spleen response or the control of inflammation-related symptoms. Also, patients with a lower burden of comorbidities at baseline who achieve a spleen response seem to have a better prognosis compared to nonresponders with a similar CCI. This cut-off of CCI $(<3)$ could be of help to identify patients who may deserve a treatment with ruxolitinib, considering that a spleen response with a limited burden of comorbid conditions may result in the best outcome. We found that patients with an increased burden of comorbidities present more frequently anemia at baseline and during therapy: physicians must be aware that a strict weekly monitoring should be carried out to check the onset of transfusion-dependence if $\mathrm{CCI}$ is higher than 3 at baseline.

Unlike in CML, BMI did not impact significantly on the response rates or on the onset of toxicities in MF patients treated with ruxolitinib. On the contrary, the drug is likely to ameliorate MF-related cachexia resulting in improved survival through the improvement of cytokine levels. 
The achievement of a spleen response in patients with initial disease-related cachexia correlated with an improvement of OS compared to patients with a similar BMI but who did not achieve such a response. Therefore, MF-related cachexia should not prevent per se the choice of starting treatment with ruxolitinib.

Our group has recently proposed a comprehensive assessment for frail patients with Philadelphia-positive and -negative chronic myeloproliferative neoplasms who need to be treated with TKIs: BMI evaluation was included together with performance status, instrumental activities of daily living, CCI and short physical performance battery (SPPB) [24]. More recently, an innovative "cachexia index" was proposed in order to provide a more objective quantification of constitutional symptoms in PMF [25]. This score takes two widely available laboratory tests into account: serum albumin and cholesterol levels, which were found to correlate with survival regardless of IPSS/DIPSS category. Overall, these parameters may reflect the hypercatabolic/cachectic state of the disease and confirm the role of the nutritional status on outcome [25].

In conclusion, comorbidities and BMI do not seem to be a contraindication for ruxolitinib therapy in MF patients, but may contribute to better define the profile of patients who deserve a strict monitoring during treatment.

\section{Ethical standards statement}

All procedures followed were in accordance with the ethical standards of the responsible committee on human experimentation (institutional and national) and with the Helsinki Declaration of 1975, as revised in 2008 (5).

\section{Statement of informed consent}

Informed consent was obtained from all patients for being included in the study. 


\section{References}

1. Saussele S, Krauss MP, Hehlmann R, et al. Impact of comorbidities on overall survival in patients with chronic myeloid leukemia: results of the randomized CML study IV. Blood 2015;126:42-49.

2. Newberry KJ, Naqvi K, Nguyen KT, et al. Comorbidities predict worse prognosis in patients with primary myelofibrosis. Cancer 2014;120:2996-3002.

3. Caan BJ, Cespedes Feliciano EM, Kroenke CH. The Importance of Body Composition in Explaining the Overweight Paradox in Cancer-Counterpoint. Cancer research 2018;78:1906-1912.

4. Obesity: preventing and managing the global epidemic. Report of a WHO consultation. World Health Organization technical report series 2000;894:i-xii, 1-253.

5. Breccia M, Loglisci G, Salaroli A, et al. Delayed cytogenetic and major molecular responses associated to increased BMI at baseline in chronic myeloid leukemia patients treated with imatinib. Cancer letters 2013;333:32-35.

6. Verstovsek S, Mesa RA, Gotlib J, et al. A double-blind, placebo-controlled trial of ruxolitinib for myelofibrosis. N Engl J Med 2012;366:799-807.

7. Cervantes F, Vannucchi AM, Kiladjian JJ, et al. Three-year efficacy, safety, and survival findings from COMFORT-II, a phase 3 study comparing ruxolitinib with best available therapy for myelofibrosis. Blood 2013;122:4047-4053.

8. Harrison CN, Vannucchi AM, Kiladjian JJ, et al. Long-term findings from COMFORT-II, a phase 3 study of ruxolitinib vs best available therapy for myelofibrosis. Leukemia 2016;30:17011707.

9. Verstovsek S, Mesa RA, Gotlib J, et al. Long-term treatment with ruxolitinib for patients with myelofibrosis: 5-year update from the randomized, double-blind, placebo-controlled, phase 3 COMFORT-I trial. J Hematol Oncol 2017;10:55.

10. Al-Ali HK, Griesshammer M, le Coutre P, et al. Safety and efficacy of ruxolitinib in an open-label, multicenter, single-arm phase $3 \mathrm{~b}$ expanded-access study in patients with myelofibrosis: a snapshot of 1144 patients in the JUMP trial. Haematologica 2016;101:1065-1073.

11. Marchetti M, Barosi G, Cervantes F, et al. Which patients with myelofibrosis should receive ruxolitinib therapy? ELN-SIE evidence-based recommendations. Leukemia 2017;31:882-888.

12. Palandri F, Palumbo GA, Bonifacio M, et al. Baseline factors associated with response to ruxolitinib: an independent study on 408 patients with myelofibrosis. Oncotarget 2017;8:7907379086.

13. Swerdlow S, Campo E, Harris N, et al. WHO Classification of Tumours of Haematopoietic and Lymphoid Tissues. Lyon, France: IARC Press 2008.

14. Barosi G, Mesa RA, Thiele J, et al. Proposed criteria for the diagnosis of post-polycythemia vera and post-essential thrombocythemia myelofibrosis: a consensus statement from the International Working Group for Myelofibrosis Research and Treatment. Leukemia 2008;22:437438 .

15. Thiele J, Kvasnicka HM, Facchetti F, et al. European consensus on grading bone marrow fibrosis and assessment of cellularity. Haematologica 2005;90:1128-1132.

16. Arber DA, Orazi A, Hasserjian R, et al. The 2016 revision to the World Health Organization classification of myeloid neoplasms and acute leukemia. Blood 2016;127:2391-2405.

17. Palandri F, Latagliata R, Polverelli N, et al. Mutations and long-term outcome of 217 young patients with essential thrombocythemia or early primary myelofibrosis. Leukemia 2015;29:13441349.

18. Tefferi A, Cervantes F, Mesa R, et al. Revised response criteria for myelofibrosis: International Working Group-Myeloproliferative Neoplasms Research and Treatment (IWG-MRT) and European LeukemiaNet (ELN) consensus report. Blood 2013;122:1395-1398.

19. Emanuel RM, Dueck AC, Geyer HL, et al. Myeloproliferative Neoplasm (MPN) Symptom Assessment Form Total Symptom Score: Prospective International Assessment of an Abbreviated Symptom Burden Scoring System Among Patients With MPNs. J Clin Oncol 2012;30:4098-4103. 
20. Charlson ME, Pompei P, Ales KL, et al. A new method of classifying prognostic comorbidity in longitudinal studies: development and validation. Journal of chronic diseases 1987;40:373-383.

21. Ording AG, Garne JP, Nystrom PM, et al. Comorbid diseases interact with breast cancer to affect mortality in the first year after diagnosis--a Danish nationwide matched cohort study. PloS one 2013;8:e76013.

22. Goede V, Cramer P, Busch R, et al. Interactions between comorbidity and treatment of chronic lymphocytic leukemia: results of German Chronic Lymphocytic Leukemia Study Group trials. Haematologica 2014;99:1095-1100.

23. Breccia M, Federico V, Loglisci G, et al. Evaluation of overall survival according to myelodysplastic syndrome-specific comorbidity index in a large series of myelodysplastic syndromes. Haematologica 2011;96:e41-42.

24. Breccia M, Palandri F, Luciano L, et al. Identification and assessment of frailty in older patients with chronic myeloid leukemia and myelofibrosis, and indications for tyrosine kinase inhibitor treatment. Annals of hematology 2018;97:745-754.

25. Nicolosi M, Penna D, Mudireddy M, et al. Development of a prognostically relevant "Cachexia Index" in Primary Myelofibrosis using serum albumin and cholesterol levels. EHA 2018, Abstract: PF614. 
Dear Editor, please find enclosed our revised paper "IMPACT OF COMORBIDITIES AND BODY MASS INDEX IN PATIENTS WITH MYELOFIBROSIS TREATED WITH RUXOLITINIB" (AOHE-D-18-00894) for Annals of Hematology journal, which has been modified according to referees' comments.

We detail below all changes made and look forward to your reply at your earliest convenience.

Best regards

Dr Massimo Breccia

Hematology

Department of Cellular Biotechnologies and Hematology

Sapienza University

Via Benevento 6, 00161 Rome, Italy

Tel. +3906857951

Fax +390644241984

e-mail: breccia@bce.uniroma1.it 


\section{Reviewer \#1}

Interesting analyses on outcome of MF patients also correlating comorbidities and BMI. Some points to discuss:

- It should be discussed if patients with MF per se should be counted in the $\mathrm{CCl}$ with 2 points (Definition leukemia: Leukemia - malignancy due to abnormal function of the bone marrow and the other blood forming organs with an increased number of immature and/or abnormal leucocytes) at least for patients with advanced phases.

We agree with the Reviewer that all patients carry the same hematological malignancy (MF); however, if we add 2 points for each patient (applying the term "leukemia" to all MF patients), the results of the present analysis would not change. Therefore, we have assigned 2 points only in case of additional malignancy prior to ruxolitinib start. This has been specified in the Material and Methods Section.

- For the BMI categories it would be interesting how the values were set. Was there any approach to have the analyses done by minimal $p$-value approach?

Minimal p-value approach was not used for the division in categories according to BMI. We stratified patients into 3 categories according to BMI, with the aim of comparing underweight, normal-weight and overweight patients. The first cut-off was however set at 21.9 in order to include the first quartile (n.96) of patients, to avoid having small numbers (only 3.2\% pts had a $B M I<18.5)$ thereby preventing substantial losses in statistical power, while the second cut-off was set at 25, in order to isolate the overweight group of patients, in compliance with the WHO stratification criteria.

This was specified in the Result Section (Impact of comorbidities and BMI on response to ruxolitinib).

- How many patients were transplanted and were they censored for survival analyses?

Overall, 17 (4.2\%). patients were submitted to allogeneic stem cell transplant after ruxolitinib failure. These patients were censored at the time of transplant for survival analysis.

This has been specified in the Result Section (Impact of comorbidities and BMI on outcome).

- The figures are difficult to read in the current outline (the differences in Grey are margial).

Ok, figures have been edited in order to improve their readability also in black and white. 


\section{Reviewer \#2}

This multicenter study analyzed the impact of comorbidities (defined according to the Charlson comorbidity index, $\mathrm{CCl}$ ) and body mass index (BMI) on responses, drug-related toxicities and outcome in a cohort of 402 patients with myelofibrosis (MF) treated with ruxolitinib. Main results showed that neither comorbidities nor BMI influence the probability of achieving a response on spleen or systemic symptoms during ruxolitinib treatment. Severe anemia, thrombocytopenia and the incidence of infections were not influenced by $\mathrm{CCl}$ and $\mathrm{BMI}$. As expected, a higher $\mathrm{CCl}$ and a lower BMI correlated with a reduced overall survival. The Authors concluded that comorbidities and BMI do not seem to be a contraindication for ruxolitinib therapy in MF patients.

Major scientific issues

1.The issue of anemia should be better addressed. Anemia was already present at ruxolinib start (tab. 1) and "patients with an increased burden of comorbidities presented more frequently anemia at baseline" (Discussion, para 2, line 7). Therefore, the statement that "comorbidities influenced the onset of ruxolitinib-related anemia (all grades) " (Abstract, results, lines 2-3 and page 4, toxicity, para 2, first line) is confusing, since anemia was already present and was not ruxolitinib-related. Accordingly, the hemoglobin level at baseline could be sufficient for 'the early identification of patients who deserve a strict monitoring during treatment' without using the $\mathrm{CCl}$; thus, the last sentence of the Abstract is futile.

In the first version of the paper, drug-induced anaemia was defined according to National Cancer Institute Common Toxicity Criteria for Adverse Events (NCI-CTCAE) v 4.0

(https://www.eortc.be/services/doc/ctc/CTCAE_4.03_2010-06-14_QuickReference_5x7.pdf).

Patients that were transfusion-dependent before the start of ruxolitinib therapy were not evaluable for drug-related anaemia. However, patients with baseline MF-related anemia (haemoglobin levels below $12 \mathrm{~g} / \mathrm{dl}$ in absence of transfusion requirement) were included in the analysis.

We agree with the Reviewer that this analysis may be confusing.

Therefore, firstly, we have repeated the analysis taking into account only drug-induced anemia, defined as increasing in anemia grade with respect to baseline levels. This analysis failed to show any impact of BMI and $\mathrm{CCl}$ on drug-induced anemia. These results are reported in the Results section and in Table 2, which has been revised.

Secondly, we have analysed the incidence of anemia only in the 132 patients that started ruxolitinib without anemia (i.e: with a haemoglobin level $\geq 12 \mathrm{~g} / \mathrm{dl}$ in absence of transfusion requirement). Here, we demonstrated that patients with $\mathrm{CCl} \geq 3$ had a higher incidence of ruxolitinib-induced grade- $\geq 2$ anemia at 3 months and at any time during therapy. Therefore, our statement that comorbidities influenced the onset of ruxolitinib-related anemia is valid for ruxolitinib-induced grade- $\geq 2$ anemia, These results are now reported in the Results section and in a new Table 3.

2. The statistical analysis reported in fig. $2 a$ and $b$ is unclear. At which comparisons the $p$ values are applied?

The $p$-values in Figures $2 a$ and $2 b$ are results of Log-rank tests and represent the overall difference between the Kaplan-Meier curves. 
We have expanded the analysis with pair-wise Log-rank tests between the curves, and the pvalues of the single log-rank pairwise survival comparisons are reported in the description of Figure 2 and confirm that the categories that significantly differ from the others (and that majorly impact on the overall difference between the categories) are represented, in Figure $\mathbf{2 a}$, by the group of patients with a spleen response and with lower CCl, that have a significantly improved Overall Survival compared to the others, and, in Figure $\mathbf{2} \boldsymbol{b}$, by the patients with no spleen response and a lower BMI, that have a significantly worse Overall Survival compared to the others.

3. Some conclusions are not supported by data, such as "The cut-off of $\mathrm{CCl}(<3)$ could be of help to identify patients who may deserve a treatment with ruxolitinib... (Discussion, para 2, lines 5-6)". Actually, the results did not show differences in the response to ruxolitinib according to $\mathrm{CCl}$.

Ok. This sentence actually referred to the result that the patients with both spleen response and $\mathrm{CCl}<3$ are projected to longer survival (Figure 2a) and may therefore represent the best target population for ruxolitinib therapy.

The sentence has been rephrased in the text and is now clearer.

Other points

1. Several repetitions should be avoided. For example, the information that 'BMI was evaluated in 380 patients' is given four times in the paper.

$O k$, repetitions have been deleted.

2. Ref. 25 should be corrected.

Ok, ref 25 has been edited. 

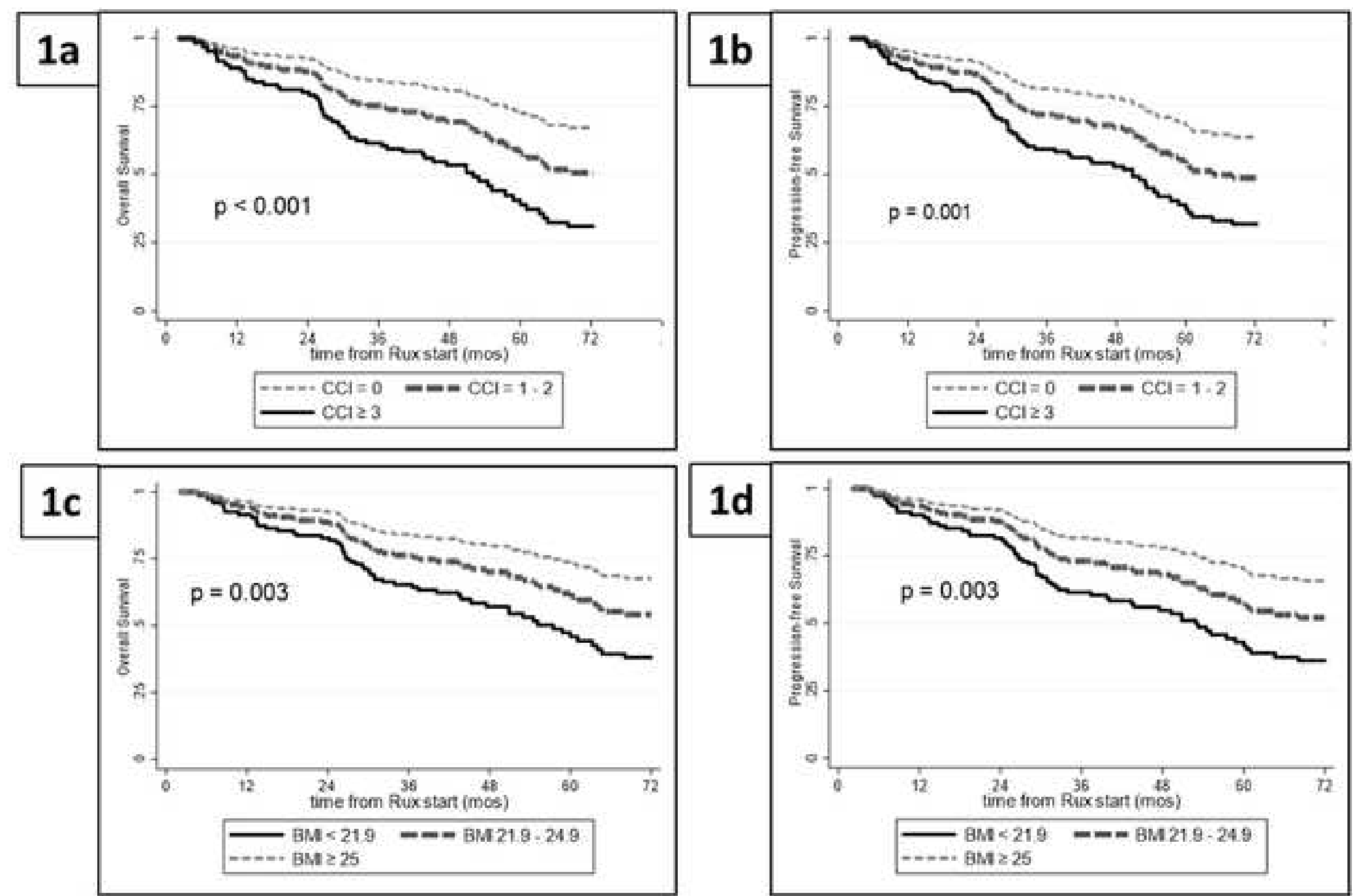

Figure 1. Overall survival (OS) and progression-free survival (PFS) according to the Charlson comorbidity index and BMI at ruxolitinib start. OS (figure 1a and 1c) and PFS (figure $1 \mathrm{~b}$ and 1d) were calculated using the Cox proportional hazards multivariate regression model with adjustment for DIPSS category at treatment start and for left truncation. 

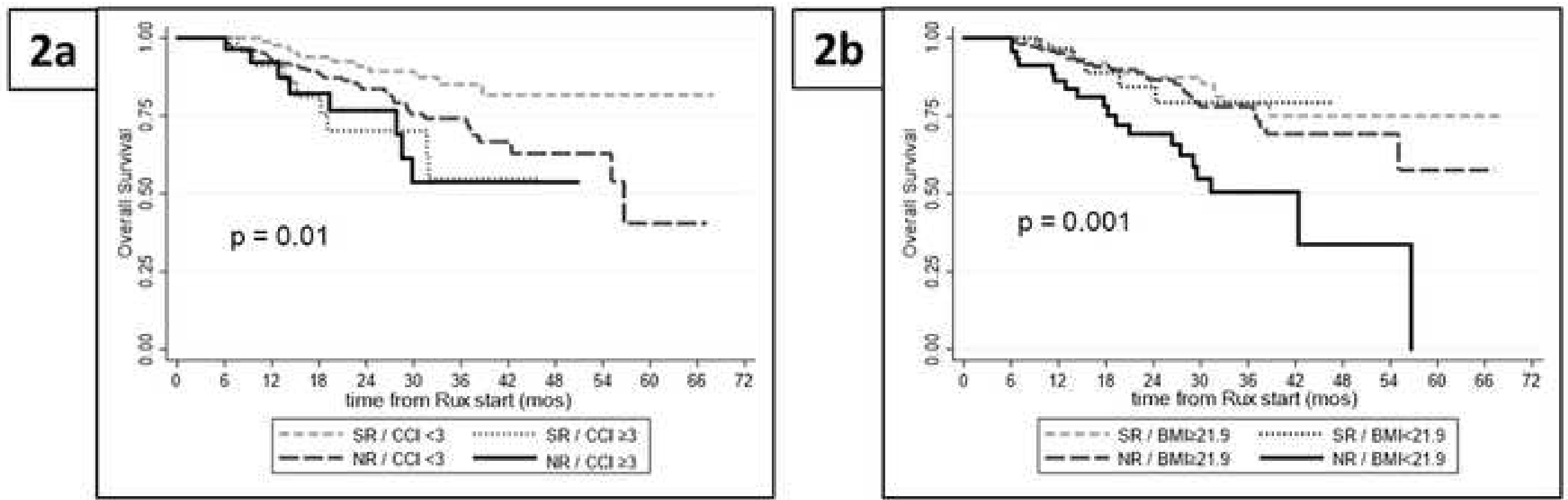

Figure 2. Landmark analysis of overall survival (OS) according to spleen response (SR) at 6 months and Charlson comorbidity index (2a) and BMI (2b) at ruxolitinib start. The p-values reported on the figures result from the Log-rank test for the overall difference between all the Kaplan-Meier curves, considering data from the 6-month landmark onwards. Pairwise Log-rank tests between the curves confirmed which categories significantly differ from the others, majorly impacting on the overall difference. Specifically, (a) patients with spleen response at 6 months and with lower CCI had a significantly improved overall survival compared to non-responding patients with a lower $\mathrm{CCI}(\mathrm{p}=0.02)$, to responding patients with a higher $\mathrm{CCI}(\mathrm{p}=0.008)$, and to non-responding patients with a higher $\mathrm{CCI}(\mathrm{p}=0.007)$, and (b) patients with no spleen response at 6 months and a lower BMI had a significantly worse overall survival compared to non-responding patients with a higher BMI $(\mathrm{p}=0.001)$, responding patients with higher BMI $(\mathrm{p}<0.001)$, and responding patients with a lower $\mathrm{BMI}$ $(\mathrm{p}=0.04)$. In both analyses, the other three categories of patients were statistically comparable. 
Table 2. Ruxolitinib-induced anemia and thrombocytopenia according to Charlson Comorbidity Index (CCI) and Body Mass Index (BMI) at ruxolitinib start. Drug-induced anemia was defined as increasing in anemia grade with respect to baseline levels. Patients that were transfusion-dependent before the start of ruxolitinib therapy were not evaluable for drug-related anaemia.

\begin{tabular}{|c|c|c|c|c|c|c|c|c|c|c|c|c|}
\hline & \multicolumn{6}{|c|}{ ANEMIA } & \multicolumn{6}{|c|}{ THROMBOCYTOPENIA } \\
\hline & $\mathrm{CCI}<3$ & $\mathrm{CCI} \geq 3$ & $p$ & $\mathrm{BMI}<21.9$ & $\mathrm{BMI} \geq 21.9$ & $p$ & $\mathrm{CCI}<3$ & $\mathrm{CCI} \geq 3$ & $p$ & $\mathrm{BMI}<21.9$ & $\mathrm{BMI} \geq 21.9$ & $p$ \\
\hline Any time & $\begin{array}{l}192 / 253 \\
(75.9 \%)\end{array}$ & $\begin{array}{l}37 / 50 \\
(74 \%)\end{array}$ & 0.78 & $\begin{array}{c}52 / 69 \\
(75.4 \%)\end{array}$ & $\begin{array}{l}172 / 224 \\
(76.8 \%)\end{array}$ & 0.8 & $\begin{array}{l}168 / 326 \\
(51.5 \%)\end{array}$ & $\begin{array}{c}39 / 76 \\
(51.3 \%)\end{array}$ & 0.97 & $\begin{array}{c}53 / 96 \\
(55.2 \%)\end{array}$ & $\begin{array}{l}145 / 284 \\
(51.1 \%)\end{array}$ & 0.48 \\
\hline At 3 months & \begin{tabular}{|l|}
$170 / 253$ \\
$(67.2 \%)$ \\
\end{tabular} & $\begin{array}{c}34 / 50 \\
(68.0 \%) \\
\end{array}$ & 0.91 & $\begin{array}{c}49 / 69 \\
(71.0 \%) \\
\end{array}$ & $\begin{array}{l}151 / 224 \\
(67.4 \%) \\
\end{array}$ & 0.57 & $\begin{array}{c}94 / 326 \\
(28.8 \%) \\
\end{array}$ & $\begin{array}{c}23 / 76 \\
(30.3 \%) \\
\end{array}$ & 0.80 & $\begin{array}{c}31 / 96 \\
(32.3 \%) \\
\end{array}$ & $\begin{array}{c}79 / 284 \\
(27.8 \%) \\
\end{array}$ & 0.40 \\
\hline At 6 months & $\begin{array}{l}124 / 232 \\
(53.45 \%)\end{array}$ & $\begin{array}{c}20 / 40 \\
(50.0 \%)\end{array}$ & 0.69 & $\begin{array}{c}32 / 62 \\
(51.6 \%)\end{array}$ & $\begin{array}{l}109 / 203 \\
(53.7 \%)\end{array}$ & 0.77 & $\begin{array}{c}92 / 326 \\
(28.2 \%)\end{array}$ & $\begin{array}{c}21 / 76 \\
(27.6 \%)\end{array}$ & 0.92 & $\begin{array}{c}25 / 96 \\
(26.0 \%)\end{array}$ & $\begin{array}{c}83 / 284 \\
(29.2 \%)\end{array}$ & 0.55 \\
\hline
\end{tabular}


Table 3. Ruxolitinib-induced anemia and thrombocytopenia according to Charlson Comorbidity Index (CCI) and Body Mass Index (BMI). Only patients with baseline haemoglobin $\geq 12 \mathrm{~g} / \mathrm{dl}$ (n. 132) and patients with baseline platelet count $\geq 150 \times 10^{9} / 1$ (n. 296) were considered evaluable.

\begin{tabular}{|c|c|c|c|c|c|c|c|c|c|c|c|c|}
\hline & \multicolumn{6}{|c|}{ ANEMIA grade $\geq 2$} & \multicolumn{6}{|c|}{ THROMBOCYTOPENIA grade $\geq 2$} \\
\hline & $\mathrm{CCI}<3$ & $\mathrm{CCI} \geq 3$ & $p$ & BMI $<21.9$ & $\mathrm{BMI} \geq 21.9$ & $p$ & $\mathrm{CCI}<3$ & $\mathrm{CCI} \geq 3$ & $p$ & BMI $<21.9$ & $\mathrm{BMI} \geq 21.9$ & $p$ \\
\hline Any time & $\begin{array}{l}40 / 116 \\
(34.5 \%)\end{array}$ & $\begin{array}{c}10 / 16 \\
(62.5 \%)\end{array}$ & 0.03 & $\begin{array}{c}12 / 27 \\
(44.4 \%)\end{array}$ & $\begin{array}{c}35 / 99 \\
(35.4 \%)\end{array}$ & 0.39 & $\begin{array}{c}34 / 244 \\
(13.9 \%)\end{array}$ & $\begin{array}{c}9 / 52 \\
(17.3 \%)\end{array}$ & 0.53 & $\begin{array}{c}12 / 67 \\
(17.9 \%)\end{array}$ & $\begin{array}{c}30 / 217 \\
(13.8 \%)\end{array}$ & 0.41 \\
\hline At 3 months & $\begin{array}{l}32 / 116 \\
(27.6 \%)\end{array}$ & $\begin{array}{c}9 / 16 \\
(56.3 \%)\end{array}$ & 0.02 & $\begin{array}{c}10 / 27 \\
(37.0 \%)\end{array}$ & $\begin{array}{c}29 / 99 \\
(29.3 \%)\end{array}$ & 0.44 & $\begin{array}{l}10 / 244 \\
(4.1 \%)\end{array}$ & $\begin{array}{c}2 / 52 \\
(3.8 \%)\end{array}$ & 0.92 & $\begin{array}{c}3 / 67 \\
(4.48 \%)\end{array}$ & $\begin{array}{l}8 / 215 \\
(3.7 \%)\end{array}$ & 0.78 \\
\hline At 6 months & $\begin{array}{l}24 / 108 \\
(22.2 \%)\end{array}$ & $\begin{array}{c}4 / 12 \\
(33.3 \%)\end{array}$ & 0.39 & $\begin{array}{c}6 / 24 \\
(25.0 \%)\end{array}$ & $\begin{array}{c}21 / 92 \\
(22.8 \%)\end{array}$ & 0.82 & $\begin{array}{l}13 / 217 \\
(6.0 \%)\end{array}$ & $\begin{array}{c}3 / 45 \\
(6.7 \%)\end{array}$ & 0.86 & $\begin{array}{c}5 / 60 \\
(8.3 \%)\end{array}$ & $\begin{array}{l}11 / 195 \\
(5.6 \%)\end{array}$ & 0.45 \\
\hline
\end{tabular}

